\title{
Emittance preserving thin film plasma mirrors for GeV scale laser plasma accelerators
}

\author{
A. Zingale $\odot,{ }^{1,}{ }^{*}$ N. Czapla, ${ }^{1}$ D. M. Nasir, ${ }^{1}$ S. K. Barber, ${ }^{2}$ J. H. Bin, ${ }^{2, \dagger}$ A. J. Gonsalves, ${ }^{2}$ F. Isono, ${ }^{2}$ \\ J. van Tilborg, ${ }^{2}$ S. Steinke, ${ }^{2,}$ K. Nakamura $\odot,{ }^{2}$ G. E. Cochran, ${ }^{3}$ J. Purcell $\odot,{ }^{1,8}$ W. P. Leemans, ${ }^{2 \|}$ \\ C. G. R. Geddes, ${ }^{2}$ C. B. Schroeder, ${ }^{2}$ E. Esarey, ${ }^{2}$ and D. W. Schumacher ${ }^{1}$ \\ ${ }^{1}$ The Ohio State University, Columbus, Ohio 43210, USA \\ ${ }^{2}$ Lawrence Berkeley National Laboratory, Berkeley, California 94720, USA \\ ${ }^{3}$ Lawrence Livermore National Laboratory, 7000 East Ave, Livermore, California 94550, USA
}

(Received 6 May 2021; accepted 18 November 2021; published 2 December 2021)

\begin{abstract}
Laser-plasma accelerators (LPAs) now routinely produce electron beams with GeV energies over acceleration lengths on the order of a few centimeters. This capability and the demonstration of multistage coupling make LPAs promising for numerous applications. However, beam quality preservation in multistage accelerators remains an obstacle because of the need to separate the laser pulse from the electron beam. Plasma mirrors can be used to redirect the laser pulse, but their substrate thickness threatens to substantially degrade the electron beam emittance. Ultrathin $(\sim 20 \mathrm{~nm})$ liquid crystal (LC) plasma mirrors are an excellent candidate to address this challenge. This work investigates the robustness of thin LC plasma mirrors in the presence of capillary discharge plasma and an auxiliary heater laser. We find they can be operated $\sim 10 \mathrm{~cm}$ from the capillary exit when a heater laser is used. We then performed a normalized emittance measurement enabled using a $20 \mathrm{~nm}$ LC plasma mirror to protect electron beam optics after the LPA. The emittance contribution from scattering through the plasma mirror is calculated to be of order $100 \mathrm{~nm}$, much less than the measured emittance of $4.0 \mu \mathrm{m}$. Finally, we develop a model to calculate plasma mirror performance based on the laser polarization and intensity, and plasma mirror thickness.
\end{abstract}

DOI: 10.1103/PhysRevAccelBeams.24.121301

\section{INTRODUCTION}

Laser-plasma accelerator (LPA) technology has grown in sophistication over the past decade. A recent development was the demonstration of staged electron acceleration [1], which can facilitate higher electron beam energies and the development of compact $\mathrm{x}$-ray free electron lasers [2,3] and $\mathrm{TeV}$ electron accelerators [4]. However, these applications have stringent requirements on the emittance of the electron beam.

\footnotetext{
"To whom correspondence should be addressed. zingale.10@osu.edu.

Present address: State Key Laboratory of High Field Laser Physics and CAS Center for Excellence in Ultra-intense Laser Science, Shanghai Institute of Optics and Fine Mechanics, Chinese Academy of Sciences, Shanghai, 201800, China.

Present address: Marvel Fusion, Munich, Bavaria, Germany.

${ }^{\S}$ Present address: Michigan State University, East Lansing, Michigan, 48824.

"Present address: Deutsches Elektronen-Synchrotron DESY, 22607 Hamburg, Germany and University of Hamburg, 20148 Hamburg, Germany.

Published by the American Physical Society under the terms of the Creative Commons Attribution 4.0 International license. Further distribution of this work must maintain attribution to the author(s) and the published article's title, journal citation, and DOI.
}

In order to couple an electron beam to multiple stages of LPA, it must pass through the optics used to divert the laser pulse into and out of the LPA beamline. For many applications, the emittance degradation due to Coulomb scattering as the electron beam passes through a mirror even as thin as a few micrometers is unacceptable $[5,6]$. In addition, the long f-number required for these LPA systems makes it impractical to allow the beam to expand between stages. For example, The Berkeley Lab Laser Accelerator (BELLA) Center petawatt (PW) [7] beam is capable of $40 \mathrm{~J}$ on target with an f-number of 65, but a damage threshold of $\sim 0.35 \mathrm{~J} / \mathrm{cm}^{2}$ is typical for mirrors used in this type of system [8]. With these parameters, the laser would need to propagate for $\sim 8 \mathrm{~m}$ to expand enough to preserve the mirrors, making this approach impractical.

Plasma mirrors $[9,10]$ are optics that take advantage of ionization of the optical surface during the rising edge of the pulse to create an overdense plasma which subsequently reflects the remainder of the pulse. In the femtosecond regime, the optical quality of the mirror is approximately maintained on the timescale of the pulse, allowing for the laser beam quality to be preserved after reflection. A typical implementation uses a few mm thick glass substrate which would significantly degrade the electron beam quality. The problem is compounded in a multistage accelerator because 
of the accumulated emittance growth through at least as many plasma mirrors as there are acceleration stages.

Over the past few years, free standing liquid crystal (LC) films have successfully been applied to ultraintense laser experiments as targets and plasma mirrors [11,12]. LC plasma mirrors can also be applied to LPA's, where they offer two major advantages: they can be formed in situ with a thickness of only $20 \mathrm{~nm}$, greatly reducing emittance growth; and the technology used to produce the films is scalable to repetition rates exceeding $1 \mathrm{~Hz}$ [13]. In this paper, we report on two experiments, carried out at the BELLA Center. The first, detailed in Sec. II is a test of robustness of thin LC films in the BELLA LPA environment. The second is a normalized emittance measurement using a LC plasma mirror to protect downstream electron beam optics, covered in Sec. III.

Plasma mirrors have proven useful for high intensity beam steering and contrast enhancement, which has lead to much experimental and theoretical work on plasma mirror reflection [14-16]. The reflected beam is usually of primary interest and typical plasma mirror thicknesses are large enough that there has not been much attention given to the transmission through a plasma mirror. This work focuses on the specific application of diverting the laser to protect downstream optics, where estimating the transmitted energy becomes important. Section IV lays out a model for predicting plasma mirror transmission as a function of incident laser intensity and polarization, and plasma mirror thickness. We then compare the model prediction to measurement from the experiment in Sec. III and simulations.

\section{SURVIVABILITY TESTS}

\section{A. Experimental setup}

There are two major threats to a thin LC film when employed as a plasma mirror on an LPA beamline at BELLA. The first is blow-off plasma from the capillary [17] discharge exit and the second is damage from an auxiliary laser pulse. Both have the potential to destroy the film before it can reflect the PW pulse. The capillary is a small, gas-filled channel that is ionized by an electrical discharge, creating a preformed plasma waveguide for the PW pulse. The auxiliary laser, or heater, then prepares the plasma in the capillary for optimal guiding of the PW pulse [18]. It is a nanosecond scale, Joule level pulse which can ionize and destroy a LC film. An experiment was carried out to determine how long the films can survive in this environment, how this might depend on film thickness, and where it can be located relative to the capillary.

Figure 1 shows a schematic of the experimental setup. A nanojoule-scale, $800 \mathrm{~nm}$ probe laser was reflected off the film and imaged to a charge-coupled device camera to measure film survival. The probe was delayed relative to the timing of the capillary discharge and heater pulse.

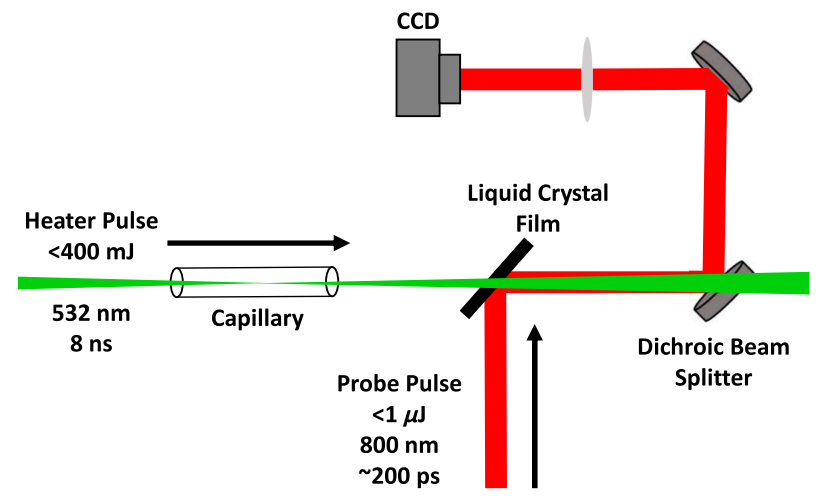

FIG. 1. A schematic of the survivability experimental setup, including the heater, capillary, and probe laser is shown.

The relative timing between the heater and capillary discharge was not changed. For the subsequent discussions, "zero delay" will correspond to the peak of the heater pulse. Negative or positive delay corresponds to times before or after the peak. The timing of the arrival of the PW pulse relative to the peak of the heater has been a subject of recent study, with evidence that optimal guiding is achieved $5 \mathrm{~ns}$ after the peak [19]. The delays were scanned in minimum step intervals of one nanosecond with the probe duration being 200 ps. Plasma mirror operation occurs on the subpicosecond timescale of the PW pulse and therefore one nanosecond after $+5 \mathrm{~ns}$ is considered more than long enough for successful plasma mirror operation. It should be noted that while plasma mirrors provide an excellent surface for reflection of short pulse lasers, they are generally considered unstable on the nanosecond timescale of the heater [20]. If a heater is required after the initial stage in a multistage LPA, it must be injected another way.

All LC films discussed in this work were made from a commonly available LC called 4-octyl-4-cyanobyphenyl, or $8 \mathrm{CB}$ for short. In this case, the films were formed using a linear slide target inserter (LSTI) [21], which provided LC targets at about once per minute in situ with target thicknesses ranging from $20 \mathrm{~nm}$ to over $1 \mu \mathrm{m}$. The film diameter was $4 \mathrm{~mm}$. The LSTI was positioned about $6 \mathrm{~cm}$ downstream of the capillary exit.

\section{B. Results}

We began with measurements of film survival in the presence of the capillary discharge alone without the heater beam. Representative probe images are shown in Fig. 2 at various delays. Films greater than about $500 \mathrm{~nm}$ in thickness survived the capillary discharge entirely. Films $\lesssim 500 \mathrm{~nm}$ were destroyed by the capillary discharge. However no film displayed any damage until about $100 \mu \mathrm{s}$. The timescale of $100 \mu \mathrm{s}$ matches well with the expected time it would take blow-off plasma to reach the film based on this distance and the speed of sound in the plasma. This is many orders of magnitude more than 


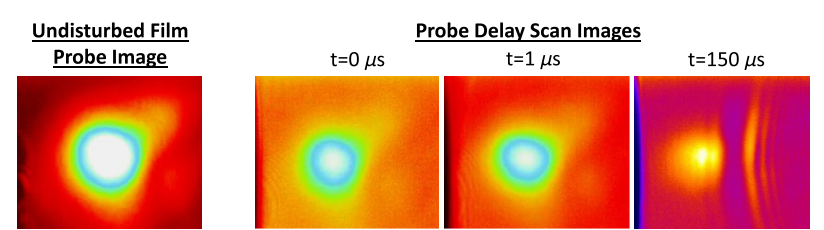

FIG. 2. These are representative probe images of $40 \mathrm{~nm}$ films at three different delay values: 0,1 , and $150 \mu$ s. An image of an undisturbed film is also displayed for comparison.

enough time considering the sub-picosecond timescale of plasma mirror operation.

Once long timescale survival of the film with the capillary discharge was confirmed, the effect of the heater beam was tested. The heater laser is a $532 \mathrm{~nm}, 8 \mathrm{~ns}$ pulse, with energy up to $400 \mathrm{~mJ}$. The average film thickness for this test was $40 \mathrm{~nm}$. Figure 3 shows a plot of the delay time at which damage was observed as a function of heater laser
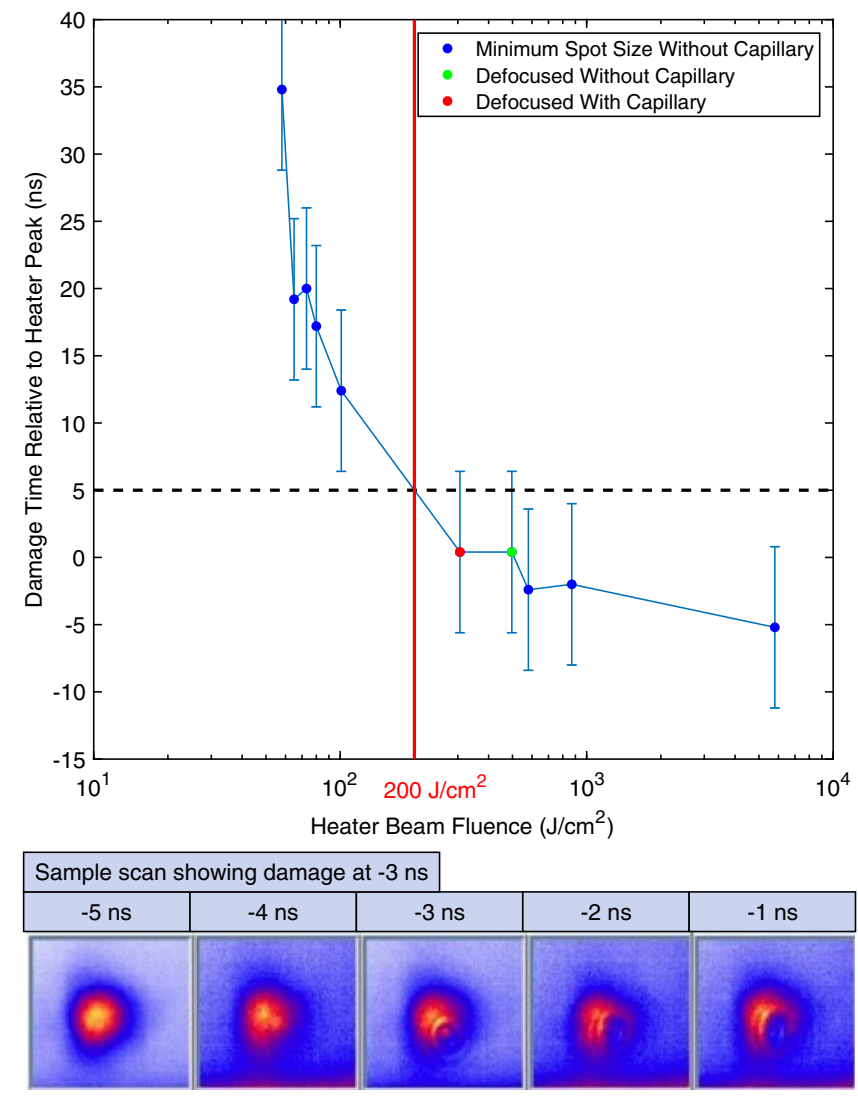

FIG. 3. Time at which damage occurred vs the heater laser fluence at the surface of the LC film. The blue points are for data collected with a focused heater beam incident on the film without a capillary present. The green point shows data collected at higher energy with a defocused heater. The Red point is a scan done with the heater and capillary discharge both active with the film $6 \mathrm{~cm}$ from the capillary exit. A representative scan is provided to show the distortions that appear when the film is damaged. The error bar represents the range of measured heater delays for a set of shots where the delay was known to be constant. fluence at the surface of the film. Each individual data point on this plot represents a delay scan over roughly 20 shots where the damage time was determined by manually inspecting the probe images for distortion. The error bar represents the maximum and minimum timing measured for a single delay setting. The source of the error was dominated by the photodiode and oscilloscope signal response. The fluence is calculated from the total energy in the heater pulse and the area associated with the full width at half maximum of the laser spot incident on the film, $\sim 100 \mu \mathrm{m}$ in the focused case, and $\sim 400 \mu \mathrm{m}$ in the defocused case. There is a clear trend showing earlier damage times for higher fluence, as one might expect. The intersection of this curve with $5 \mathrm{~ns}$ delay provides us with an estimate for the maximum tolerable fluence on the film. This is marked by the solid red line in the plot and corresponds to $\sim 200 \mathrm{~J} / \mathrm{cm}^{2}$. With a $400 \mathrm{~mJ}$ pulse and the observed divergence seen from the capillary exit, the minimum safe operating distance from the capillary exit is about $10 \mathrm{~cm}$. Accounting for the measurement error, the worst case scenario would drop the maximum tolerable fluence to $\sim 115 \mathrm{~J} / \mathrm{cm}^{2}$, requiring an extra $3 \mathrm{~cm}$ for safe distance. This shows promise for use in LPA staging, with even smaller standoff distances possible for schemes where a heater laser is not required.

\section{EMITTANCE MEASUREMENT}

\section{A. Experimental setup \\ 1. Emittance diagnostic}

The intrinsic aspects of electron beams generated by an LPA make transverse emittance characterization challenging for traditional emittance measurement methods [22]. Large energy spread and divergence impose resolution limits on standard pepper-pot methods. Substantial shot-toshot variations make multishot techniques like quadrupole scans and wire scans unreliable. Recently, however, a technique was developed which is specifically tailored for LPA emittance measurements [23].

Using a focusing element, the electron beam is imaged onto a scintillating screen after first being dispersed in energy by a magnetic dipole. The natural dependence of focusing strength on electron energy results in beam size variation at the scintillating screen. The beam size is smallest for the energy which exactly satisfies the imaging condition. The beam size at the screen increases for the lower or higher energy electrons which are over or under focused. This dependence can be well described using standard electron beam optics, which can then be used to estimate the emittance. Recently, this technique (sometimes referred to as the "bowtie" or "butterfly" method due to the characteristic shape of the imaged beam) has become one of the more popular techniques for plasma based accelerators [23-25]. 
This measurement technique was implemented on the BELLA PW beamline using an active plasma lens [26,27] (APL) for the focusing element and a pair of opposite polarity dipoles for the dispersion element. The APL that was used has a $500 \mu \mathrm{m}$ diameter, is $6 \mathrm{~cm}$ long and was filled with argon at 10 Torr fill pressure. Using a heavier gas like argon (compared to light gas species like hydrogen) has some notable advantages in terms of emittance preservation [28]. This setup enables normalized emittance measurements with a resolution limit of a few $\mu$ m for beams with central energies up to $2 \mathrm{GeV}$. Details on the design can be found in Ref. [29].

\section{Influence of plasma mirror thickness}

This diagnostic relies on the use of a plasma mirror because the APL (which typically has a diameter less than $1 \mathrm{~mm}$ ) must be placed close to the LPA target to capture the highly divergent LPA source. Without some way to separate the high intensity laser from the electron beam, the APL will quickly suffer catastrophic damage. The plasma mirror (located between the LPA source and APL) redirects the bulk of the laser energy away from the nominal propagation direction leaving the propagation direction of the electron beam itself unaffected. However, electron beams propagating through material undergo scattering which in turn can dilute the emittance. For thin films, the effect on the electron beam is most strongly related to Coulomb scattering which is well understood [6]. A previous experiment with a similar setup described in Ref. [27] used a $\sim 20 \mu \mathrm{m}$ thick, tape-based plasma mirror [30]. The emittance growth due to scattering in the plasma mirror was the dominant contribution to the final emittance measurement in that case, limiting the resolution of the measurement.

Using a replenishable, $\sim 20 \mathrm{~nm}$, LC plasma mirror instead offers much improvement. Following Ref. [6], we can estimate the electron beam emittance growth due to scattering in the plasma mirror. It is a function of accelerator geometry, source parameters, and the plasma mirror material properties and thickness. In the case of this experiment, the $20 \mathrm{~nm} \mathrm{LC}$ plasma mirror was placed $11 \mathrm{~cm}$ downstream of the LPA. Assuming an initial divergence and emittance, we can propagate the electron beam from the source to the plasma mirror and then calculate the emittance after the plasma mirror.

The output normalized emittance is plotted in Fig. 4 as a function of the normalized emittance input to the plasma mirror for both $20 \mathrm{~nm} \mathrm{LC}$ (used in this experiment) and $20 \mu \mathrm{m}$ Kapton. These curves illustrate the effects of scattering through the plasma mirror for a range of initial parameters. For low-input emittance, the scattering contribution is dominant and the output emittance is nearly independent of the input. In this low-emittance regime, the plasma mirror material and thickness and electron beam divergence are critical. But regardless of the initial

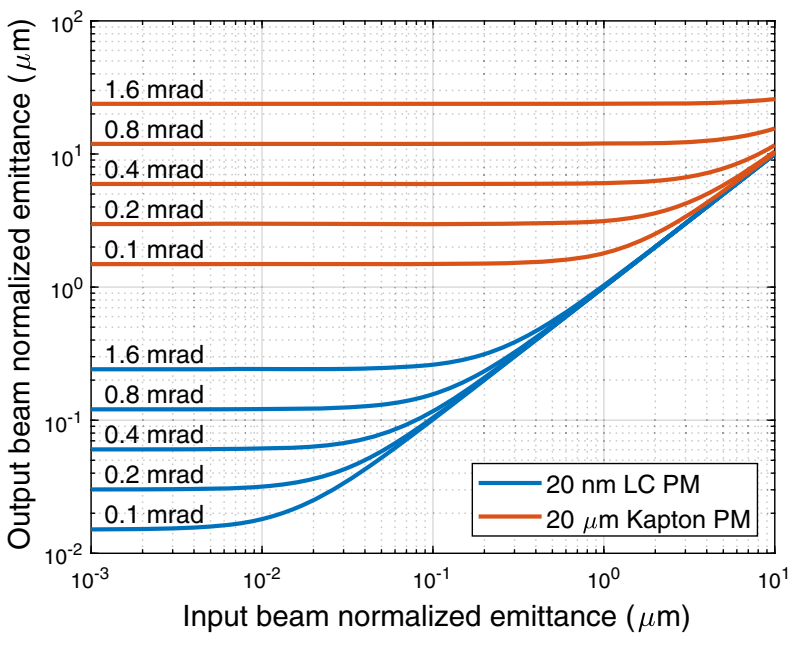

FIG. 4. Graph of normalized emittance after transmission through a plasma mirror as a function of initial normalized emittance. All curves are calculated using the method described in Ref. [6]. The effect of scattering through two different types of plasma mirror (20 nm LC and $20 \mu \mathrm{m}$ Kapton) is compared for five discrete initial divergence values. Initial divergence is labeled on each curve.

divergence, the LC plasma mirrors offer two orders of magnitude improvement over the more conventional thin plasma mirror. For the $\sim 0.5 \mathrm{mrad}$ divergence electron beams expected, the minimum output emittance improves from nearly $10 \mu \mathrm{m}$ to less than $0.1 \mu \mathrm{m}$. This may be low enough for other possible sources of emittance growth in the system such as scattering from background ions in the APL plasma to become important for measurement resolution [31]. However, if the input emittance is high enough, the blue and orange curves merge, indicating that the plasma mirror contribution is insignificant, and the material and thickness no longer matter. For this accelerator geometry, the transition between these regimes is in the few $\mu \mathrm{m}$ range, below which only the ultrathin LC can preserve the input emittance.

\section{B. Results}

Electron beams are generated using the BELLA PW laser system which is focused into a gas cell filled with a mixture of helium (99\%) and nitrogen (1\%). The laser is operated in a "low power" mode using only $10 \mathrm{~J}$ for a peak power of $0.25 \mathrm{PW}$. The produced beams are quasimonoenergetic with an average energy of $0.84 \mathrm{GeV}$ and an energy spread of $30 \%$. Upon emerging from the gas cell, the electron beam copropagates with the laser pulse to a $20 \mathrm{~nm}$ LC plasma mirror which diverts the laser pulse. The electron beam is then focused and dispersed by the aforementioned APL and magnetic dipoles. Figure 5(a) shows an image of an electron beam profile at the scintillating screen. From this image, we determine the beam size as a function of energy (Fig. 5(d) green line) 

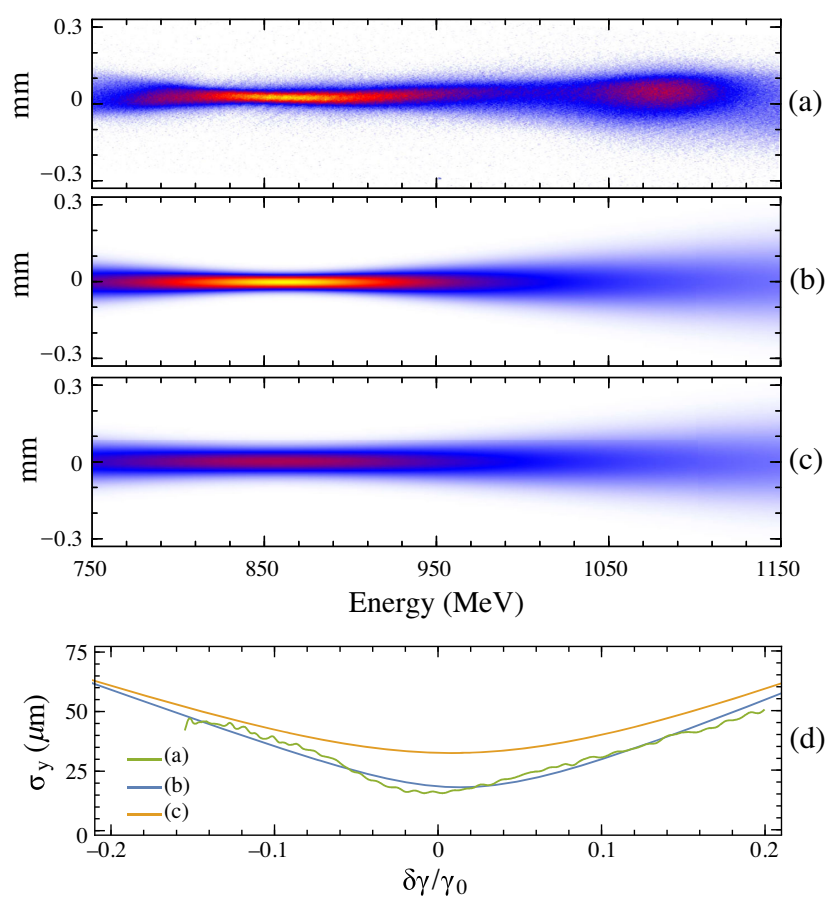

plasma mirror needs to be well understood. This is in contrast to plasma mirror operation for pulse contrast enhancement where the reflected beam is paramount. We have developed a new model for predicting both reflection and transmission from the plasma mirror as a function of incident intensity, polarization, and plasma mirror thickness. These need not sum to unity due to absorption. A few assumptions are made to simplify the calculation. First, we treat the LC plasma mirror as a carbon plasma slab with thickness equal to the initial known thickness of the LC film. The electron density is calculated by estimating the average ionization state of the carbon based on the appearance intensity of carbon ions along with the density of the LC. Due to the typical intensity range of operation of plasma mirrors $\left(10^{15}-10^{17} \mathrm{~W} / \mathrm{cm}^{2}\right)$, we chose an ionization of $Z=4$ or $\mathrm{C}^{4+}[32,33]$. For $8 \mathrm{CB}$, this works out to an electron number density $n_{e}=2.4 \times 10^{23} \mathrm{~cm}^{-3}$ or $137 n_{c}$, where $n_{c}$ is the critical density at $800 \mathrm{~nm}$. Second, for purposes of this model, we do not expect any significant expansion before or during the interaction because of the ultrashort pulse duration, so the density is treated as a constant. Finally, the incident intensity is treated as constant, corresponding to the average intensity of the pulse. This is a reasonable simplification because the reflectance is not a strong function of intensity. It is shown below that the reflectance only varies by $\sim 10 \%$ over an entire order of magnitude in intensity.

With these assumptions, we can use the Fresnel equations for fractional reflection and transmission [34] at an interface with complex index of refraction of the plasma to find the overall reflection and transmission through the thin plasma slab. We now turn to calculation of the complex index of refraction, $\hat{n}$. This is done by way of the Drude model [34], which yields,

$$
\hat{n}=n(1+i k),
$$

beam transport using source parameters determined from the above fit. The result of the simulation is shown in Fig. 5(b). The simulation includes the scattering effect from a $20 \mathrm{~nm}$ LC film. From the simulation, the energy dependent beam size is retrieved and found to be indistinguishable from the original fit of the measured trace (Fig. 5(d) blue line), which indicates the negligible impact of the LC plasma on the beam emittance. We repeated the simulation in Fig. 5(c), instead using a $20 \mu \mathrm{m}$ plasma mirror. The result is noticeably different from the $20 \mathrm{~nm}$ case. For this case, the energy dependent beam size (Fig. 5(d) yellow line) is substantially different and represents an emittance of $6.8 \mu \mathrm{m}$, a $70 \%$ increase from the measured value.

\section{ANALYTICAL MODEL OF LC PLASMA MIRROR PERFORMANCE}

When employing a plasma mirror to protect downstream components in the beamline, the transmission through the

where

$$
\begin{gathered}
n=\left[\frac{1-\alpha}{2}+\frac{1}{2} \sqrt{(\alpha-1)^{2}+\left(\frac{\alpha \nu_{e i}}{\omega_{L}}\right)^{2}}\right]^{\frac{1}{2}}, \\
k=\frac{\alpha \nu_{e i}}{2 \omega_{L} n^{2}},
\end{gathered}
$$

and $\alpha$ is defined as

$$
\alpha \equiv \frac{\omega_{p}^{2}}{\omega_{L}^{2}+\nu_{e i}^{2}} .
$$

where $\omega_{p}$ is the plasma frequency, $\omega_{L}$ is the laser frequency, and $\nu_{e i}$ is the Spitzer collision rate [35]. We now require the temperature in order to calculate the Spitzer rate because $\nu_{e i}=\frac{4 \sqrt{2 \pi}}{3} n_{e} Z e^{4} m_{e}^{-1 / 2} T_{e}^{-3 / 2} \ln \Lambda$. In this 
expression, $n_{e}$ is the electron number density, $e$ and $m_{e}$ are the electron charge and mass, $\ln \Lambda$ is the Coulomb logarithm, and $T_{e}$ is the electron temperature. To calculate this temperature, we use the model derived by Gibbon [36], following the work of Rozmus and Tikhonchuk [37], for the front surface temperature in the intensity and pulse duration regime for LPA. This model is derived from an energy balance that takes into account thermal transport from the front surface into the bulk of the plasma. The resulting expression for the electron temperature is

$$
T_{e}=1.35\left(\frac{\sqrt{2 m_{e}} \pi^{3 / 2} e^{4} \eta^{2} I_{0}^{2} Z t \ln \Lambda}{48 n_{e}}\right)^{2 / 9},
$$

where $\eta$ is the absorption fraction, $I_{0}$ is the incident intensity, $Z$ is the ionization, $n_{e}$ is the electron density, and $t$ is the pulse duration. This leads us to a possible inconsistency because the temperature depends on the absorption but the absorption follows directly from the transmittance and reflectance. In order to treat the absorption self consistently, we take an iterative approach that ensures the final calculated value for the absorption matches the value used to compute the temperature. Figure 6 shows a flow chart that describes the approach. It starts with a reasonable guess for the absorption, expressed as a fraction between zero and unity, $\eta_{0}$, which is used to estimate the front surface temperature. Using the Drude model and the Spitzer collision rate, the complex index of refraction of the plasma follows. From the index, the reflectance and transmittance are calculated using the Fresnel equations. Finally, conservation of energy then leads to the absorption fraction, $\eta_{m}$, where $m$ denotes the number of iterations. $\eta_{m}$ is fed back into the algorithm on the $(m+1)^{\text {th }}$ iteration until $\eta$ converges. The absorption is considered to be sufficiently converged if $\left|\eta_{m}-\eta_{m-1}\right| \leq$ 0.001 . Typically, convergence is achieved after only three or four iterations.

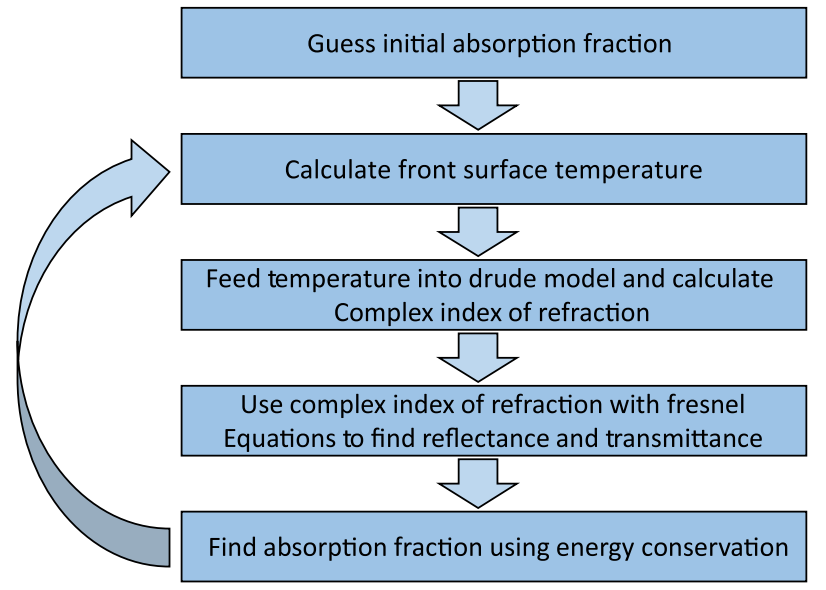

FIG. 6. A schematic overview of algorithm used to calculate reflection, transmission, and absorption is shown.
To validate this model, we compare to particle-in-cell (PIC) simulations of pulse-cleaning plasma mirror operation developed in Ref. [16]. Briefly, 2D3V implicit simulations were performed using the PIC code large scale plasma [38] for s-polarized, $800 \mathrm{~nm}$ laser pulses incident on LC plasma mirrors at $16 \mathrm{deg}$ angle of incidence for a range of intensities. The plasma mirrors were modeled as thin slabs of initially neutral hydrogen and carbon and ionized dynamically using a Monte Carlo multiphoton ionization model. Collisions were modeled using a capped Spitzer rate. Multiple two-dimensional simulations were combined to model a three-dimensional laser spot. The cell size was $2.5 \times 3.0 \mathrm{~nm}$ and the time step was chosen for a Courant ratio of 0.75 . Finally, the simulations used 10 macroparticles per species per cell. These simulations were validated with experimental reflectance data in Fig. 6 of Ref. [12].
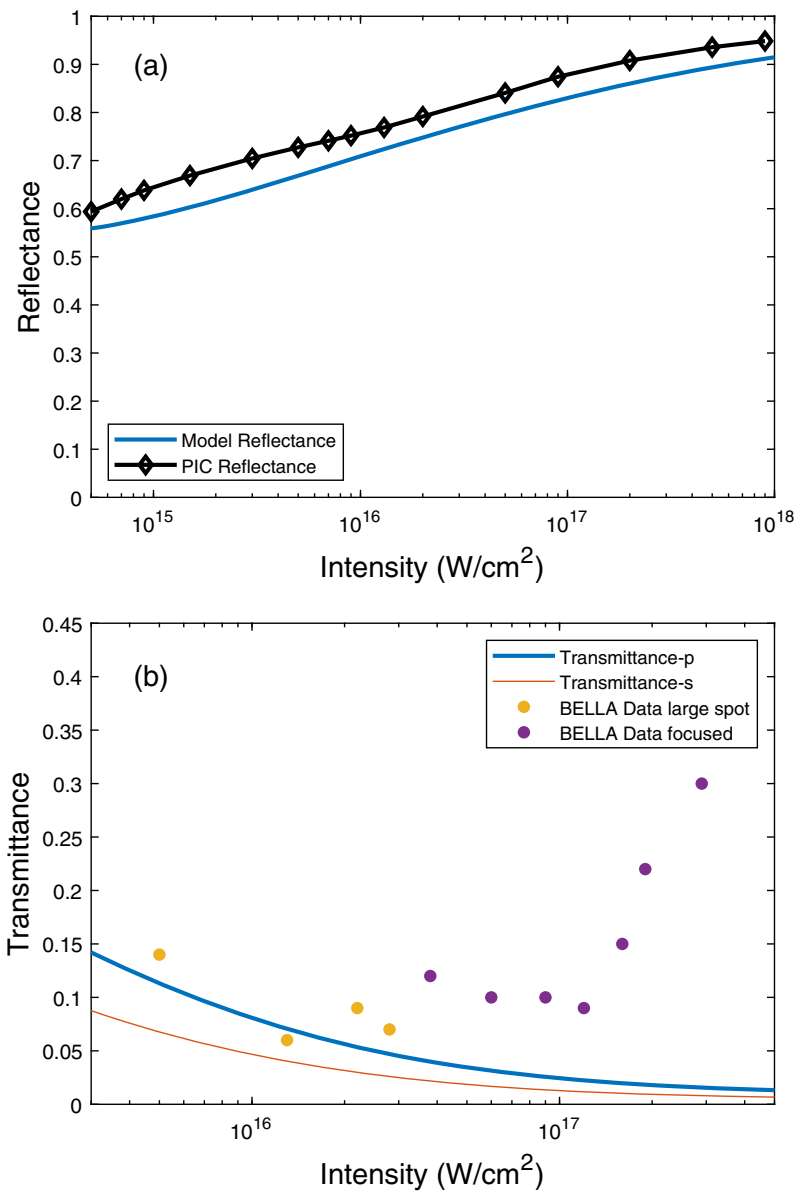

FIG. 7. (a) Reflectance vs intensity for s-polarization at $15^{\circ}$ incidence as calculated by the model described in this article (blue) is shown in comparison to simulations from Ref. [16] (black). (b) Transmittance vs intensity for both s (solid orange curve) and $\mathrm{p}$ (solid blue curve) polarizations at $45^{\circ}$ incidence again calculated with the model described here. These curves are compared to the transmission data taken in the experiment in Sec. III with a focused (purple circles) and defocused (yellow circles) beam incident on the plasma mirror. 
Figure 7(a) shows a plot of the simulation results for a $270 \mathrm{~nm}$ plasma mirror along with the predictions given by the model described above for the same conditions. We see that they match to within a few percent over more than three orders of magnitude in intensity. However, the assumptions necessary for this calculation limit the intensity range over which the model can be applied. At lower intensity, the Spitzer collision frequency becomes unphysically high, leading to an unrealistic index of refraction. Practically, this results in a loss of convergence of the model around $2 \times 10^{14} \mathrm{~W} / \mathrm{cm}^{2}$. In the high-intensity case, the temperature and therefore plasma soundspeed can become large enough that there is significant expansion during the laser pulse duration. For instance, near $10^{17} \mathrm{~W} / \mathrm{cm}^{2}$, the expected expansion is about $20 \mathrm{~nm}$, the same as the initial thickness of the plasma mirror, leading to a density drop greater than $2 \times$. This presents a problem for the model because constant density is assumed, as well as the implicit assumption of a step-like density profile through the use of the Fresnel relations.

However, there is no clear upper intensity limit where the model breaks down, as evidenced by the agreement with the simulations plotted in Fig. 7(a) all the way up to $10^{18} \mathrm{~W} / \mathrm{cm}^{2}$, even though the simulations account for expansion during the laser pulse. But the experimental data in Ref. [12] show that near an intensity of $2 \times$ $10^{16} \mathrm{~W} / \mathrm{cm}^{2}$ the reflectance begins to fall sharply. This drop in reluctance above some critical intensity has been commonly observed in prior plasma mirror studies [14,15]. Neither the simulation nor the analytical model capture this behavior, suggesting that the simplifications made in the model that are accounted for in the simulations are not the dominant cause of the disagreement with experiment. However, neither model accounts for any pre-expansion due to the slow turn on of the pulse typically referred to as the pedestal. The pedestal will initiate expansion of the plasma mirror before the arrival of the main pulse and lower the peak intensity for which the model is accurate. Previous work has also suggested that this can be an issue [30].

Qualitatively, this effect is important when considering the use of a plasma mirror to inject a laser pulse into a LPA. The peak reflectivity can be controlled somewhat by design parameters such as polarization, and incidence angle, and spot size, but there will be a limit [12]. Any application using plasma mirrors for this purpose would have to account for this loss in energy in the laser design.

Figure 7(b) compares values obtained from this model to new transmittance results measured in the experiment described above. There is good agreement for the defocused case and an underestimate for the lower energy focused beam for intensities below $\sim 10^{17} \mathrm{~W} / \mathrm{cm}^{2}$. Above this intensity, we see a rise in transmission that is not predicted by the model. Like the fall in reflectance noted above, this could be due to increased heating and expansion from the pedestal. Ongoing experimental and theoretical investigations to fully understand the cause of this behavior will be the subject of a future publication. It also should be noted that, as one might expect, the s-polarized case leads to a reduction in transmittance. The ability to predict and quantify plasma mirror performance with this relatively simple analytical calculation will aid in future experimental design.

\section{CONCLUSIONS}

We have shown the specific conditions under which LC films of order $20 \mathrm{~nm}$ thickness can be used as plasma mirrors near a LPA. Films withstand the blowout plasma from a capillary discharge for many microseconds. If a heater laser pulse is used to prepare the plasma, the fluence incident on the film must not exceed $\sim 200 \mathrm{~J} / \mathrm{cm}^{2}$ in order for the film to survive long enough to reflect the main pulse. This corresponds to a standoff distance from the capillary of $10 \mathrm{~cm}$ for the specific heater laser divergence and energy tested. The plasma mirrors are capable of rejecting up to 94\% of the incident laser pulse. The protection of downstream optics in close proximity to the LPA, combined with the reduced thickness, enabled a normalized emittance measurement of LPA electron beams. The scattering of electrons through the plasma mirror contributed a negligible amount to the measured value of $4.0_{-1.0}^{+1.3} \mu \mathrm{m}$. We have also introduced a simple model to compute the reflected, transmitted, and absorbed laser energy from ultrathin plasma mirrors in the intensity range of $\sim 10^{14}-\sim 10^{17} \mathrm{~W} / \mathrm{cm}^{2}$.

\section{ACKNOWLEDGMENTS}

This work was supported by the U.S. Department of Energy (DOE), Office of Science, Offices of High Energy Physics and Basic Energy Sciences, under Contract No. DE-AC02-05CH11231, the DOE Office of Science, Fusion Energy Sciences under Contract No. DESC0018192, the LaserNetUS initiative.

[1] S. Steinke, J. van Tilborg, C. Benedetti, C. G. R. Geddes, C. B. Schroeder, J. Daniels, K. K. Swanson, A. J. Gonsalves, K. Nakamura, N. H. Matlis, B. H. Shaw, E. Esarey, and W.P. Leemans, Multistage coupling of independent laser-plasma accelerators, Nature (London) 530, 190 (2016).

[2] K. Nakajima, Towards a table-top free-electron laser, Nat. Phys. 4, 92 (2008).

[3] M. Fuchs, R. Weingartner, A. Popp, Z. Major, S. Becker, J. Osterhoff, I. Cortrie, B. Zeitler, R. Hrlein, G. D. Tsakiris, U. Schramm, T. P. Rowlands-Rees, S. M. Hooker, D. Habs, F. Krausz, S. Karsch, and F. Grner, Laser-driven soft-X-ray undulator source, Nat. Phys. 5, 826 (2009).

[4] W. Leemans and E. Esarey, Laser-driven plasma-wave electron accelerators, Phys. Today 62, No. 3, 44 (2009). 
[5] P. Emma et al., First lasing and operation of an angstromwavelength free-electron laser, Nat. Photonics 4, 641 (2010).

[6] M. B. Reid, Electron beam emittance growth in thin foils: A betatron function analysis, J. Appl. Phys. 70, 7185 (1991).

[7] K. Nakamura, H. Mao, A. J. Gonsalves, H. Vincenti, D. E. Mittelberger, J. Daniels, A. Magana, C. Toth, and W. P. Leemans, Diagnostics, control and performance parameters for the bella high repetition rate petawatt class laser, IEEE J. Quantum Electron. 53, 1 (2017).

[8] M. Streeter, P. Foster, T. Winstone, B. Parry, and P. Rajeev, Damage testing of reflective coatings in Astra TA2 (2007).

[9] B. Dromey, S. Kar, M. Zepf, and P. Foster, The plasma mirrora subpicosecond optical switch for ultrahigh power lasers, Rev. Sci. Instrum. 75, 645 (2004).

[10] G. Doumy, F. Quéré, O. Gobert, M. Perdrix, P. Martin, P. Audebert, J. C. Gauthier, J.-P. Geindre, and T. Wittmann, Complete characterization of a plasma mirror for the production of high-contrast ultraintense laser pulses, Phys. Rev. E 69, 026402 (2004).

[11] P. L. Poole, L. Obst, G. E. Cochran, J. Metzkes, H.-P. Schlenvoigt, I. Prencipe, T. Kluge, T. Cowan, U. Schramm, D. W. Schumacher, and K. Zeil, Laser-driven ion acceleration via target normal sheath acceleration in the relativistic transparency regime, New J. Phys. 20, 013019 (2018).

[12] P. L. Poole, A. Krygier, G. E. Cochran, P. S. Foster, G. G. Scott, L. A. Wilson, J. Bailey, N. Bourgeois, C. Hernandez-Gomez, D. Neely, P. P. Rajeev, R. R. Freeman, and D. W. Schumacher, Experiment and simulation of novel liquid crystal plasma mirrors for high contrast, intense laser pulses, Sci. Rep. 6, 32041 (2016).

[13] D. Schumacher, P. Poole, C. Willis, G. Cochran, R. Daskalova, J. Purcell, and R. Heery, Liquid crystal targets and plasma mirrors for laser based ion acceleration, J. Instrum. 12, C04023 (2017).

[14] A.S. Pirozhkov et al., Diagnostic of Laser Contrast Using Target Reflectivity, Appl. Phys. Lett. 94, 241102 (2009).

[15] G. G. Scott, V. Bagnoud, C. Brabetz, R. J. Clarke, J. S. Green, R. I. Heathcote, H. W. Powell, B. Zielbauer, T. D. Arber, P. McKenna, and D. Neely, Optimization of plasma mirror reflectivity and optical quality using double laser pulses, New J. Phys. 17, 033027 (2015).

[16] G. E. Cochran, P. L. Poole, and D. W. Schumacher, Modeling pulse-cleaning plasma mirrors from dielectric response to saturation: A particle-in-cell approach, Phys. Plasmas 26, 103103 (2019).

[17] W. P. Leemans, B. Nagler, A. J. Gonsalves, C. Tóth, K. Nakamura, C. G. R. Geddes, E. Esarey, C. B. Schroeder, and S. M. Hooker, GeV electron beams from a centimetrescale accelerator, Nat. Phys. 2, 696 (2006).

[18] A. J. Gonsalves et al., Petawatt Laser Guiding and Electron Beam Acceleration to $8 \mathrm{GeV}$ in a Laser-Heated Capillary Discharge Waveguide, Phys. Rev. Lett. 122, 084801 (2019).

[19] A. J. Gonsalves et al., Laser-heated capillary discharge plasma waveguides for electron acceleration to $8 \mathrm{gev}$, Phys. Plasmas 27, 053102 (2020).
[20] C. Thaury, F. Qur, J.-P. Geindre, A. Levy, T. Ceccotti, P. Monot, M. Bougeard, F. Rau, P. dOliveira, P. Audebert, R. Marjoribanks, and P. Martin, Plasma mirrors for ultrahighintensity optics, Nat. Phys. 3, 424 (2007).

[21] P. L. Poole, C. Willis, G. E. Cochran, R. T. Hanna, C. D. Andereck, and D. W. Schumacher, Moderate repetition rate ultra-intense laser targets and optics using variable thickness liquid crystal films, Appl. Phys. Lett. 109, 151109 (2016).

[22] A. Cianchi, M. Anania, M. Bellaveglia, M. Castellano, E. Chiadroni, M. Ferrario, G. Gatti, B. Marchetti, A. Mostacci, R. Pompili, C. Ronsivalle, A. Rossi, and L. Serafini, Challenges in plasma and laser wakefield accelerated beams diagnostic, Nucl. Instrum. Methods Phys. Res., Sect. A 720, 153 (2013).

[23] R. Weingartner, S. Raith, A. Popp, S. Chou, J. Wenz, K. Khrennikov, M. Heigoldt, A. R. Maier, N. Kajumba, M. Fuchs, B. Zeitler, F. Krausz, S. Karsch, and F. Grüner, Ultralow emittance electron beams from a laser-wakefield accelerator, Phys. Rev. ST Accel. Beams 15, 111302 (2012).

[24] N. Vafaei-Najafabadi, W. An, C. E. Clayton, C. Joshi, K. A. Marsh, W. B. Mori, E. C. Welch, W. Lu, E. Adli, J. Allen, C. I. Clarke, S. Corde, J. Frederico, S. J. Gessner, S. Z. Green, M. J. Hogan, M. D. Litos, and V. Yakimenko, Evidence for high-energy and low-emittance electron beams using ionization injection of charge in a plasma wakefield accelerator, Plasma Phys. Controlled Fusion 58, 034009 (2016).

[25] S. K. Barber, J. van Tilborg, C. B. Schroeder, R. Lehe, H.-E. Tsai, K. K. Swanson, S. Steinke, K. Nakamura, C. G. R. Geddes, C. Benedetti, E. Esarey, and W. P. Leemans, Measured Emittance Dependence on the Injection Method in Laser Plasma Accelerators, Phys. Rev. Lett. 119, 104801 (2017).

[26] W. K. H. Panofsky and W. R. Baker, A focusing device for the external $350 \mathrm{mev}$ proton beam of the 184 inch cyclotron at berkeley, Rev. Sci. Instrum. 21, 445 (1950).

[27] J. van Tilborg, S. Steinke, C. G. R. Geddes, N. H. Matlis, B. H. Shaw, A. J. Gonsalves, J. V. Huijts, K. Nakamura, J. Daniels, C. B. Schroeder, C. Benedetti, E. Esarey, S. S. Bulanov, N. A. Bobrova, P. V. Sasorov, and W. P. Leemans, Active Plasma Lensing for Relativistic Laser-PlasmaAccelerated Electron Beams, Phys. Rev. Lett. 115, 184802 (2015).

[28] C. A. Lindstrøm, E. Adli, G. Boyle, R. Corsini, A. E. Dyson, W. Farabolini, S. M. Hooker, M. Meisel, J. Osterhoff, J.-H. Röckemann, L. Schaper, and K. N. Sjobak, Emittance Preservation in an Aberration-Free Active Plasma Lens, Phys. Rev. Lett. 121, 194801 (2018).

[29] S. K. Barber, J. H. Bin, A. J. Gonsalves, F. Isono, J. van Tilborg, S. Steinke, K. Nakamura, A. Zingale, N. A. Czapla, D. Schumacher, C. B. Schroeder, C. G. R. Geddes, W. P. Leemans, and E. Esarey, A compact, high resolution energy, and emittance diagnostic for electron beams using active plasma lenses, Appl. Phys. Lett. 116, 234108 (2020).

[30] B. Shaw, S. Steinke, J. van Tilbrog, and W. P. Leemans, Reflectance characterization of tape-based plasma mirrors, Phys. Plasmas 23, 063118 (2016). 
[31] C. B. Schroeder, E. Esarey, C. G. R. Geddes, C. Benedetti, and W.P. Leemans, Physics considerations for laserplasma linear colliders, Phys. Rev. ST Accel. Beams 13, 101301 (2010).

[32] N. B. Delone and V. P. Krainov, Tunneling and barriersuppression ionization of atoms and ions in a laser radiation field, Phys. Usp. 41, 469 (1998).

[33] A. Kramida, Yu. Ralchenko, and J. Reader (NIST ASD Team), NIST Atomic Spectra Database (ver. 5.7.1), https:// physics.nist.gov/asd.

[34] M. Born, E. Wolf, A. B. Bhatia, P. C. Clemmow, D. Gabor, A. R. Stokes, A. M. Taylor, P. A. Wayman, and W. L. Wilcock, Principles of Optics: Electromagnetic Theory of
Propagation, Interference and Diffraction of Light, 7th ed. (Cambridge University Press, Cambridge, England, 1999).

[35] L. Spitzer, Physics of fully ionized gases, Am. J. Phys. 31, 890 (1963).

[36] P. Gibbon, Short Pulse Laser Interactions with Matter (World Scientific Publishing Company, Singapore, 2004).

[37] W. Rozmus and V. T. Tikhonchuk, Skin effect and interaction of short laser pulses with dense plasmas, Phys. Rev. A 42, 7401 (1990).

[38] D. Welch, D. Rose, R. Clark, T. Genoni, and T. Hughes, Implementation of an non-iterative implicit electromagnetic field solver for dense plasma simulation, Comput. Phys. Commun. 164, 183 (2004). 